\title{
Algorithm of Iterative Process for Some Mappings and Iterative Solution of Some Diffusion Equation
}

\author{
Liu Wenjun \\ Department of Mathematics \\ Jiujiang University \\ Jiujiang , China \\ liuwj4573@163.com
}

\author{
Meng Jinghua \\ Department of Mathematics \\ Jiujiang University \\ Jiujiang, China \\ mengjh1956@sina.com
}

\begin{abstract}
In Hilbert spaces, through improving some corresponding conditions in some literature and extending some recent relevent results, a strong convergence theorem of some implicit iteration process for pesudocon-traction mappings and explicit iteration process for nonexpansive mappings were established. And by using the result, some iterative solution for some equation of response diffusion were obtained.
\end{abstract}

Keywords - pesudocon-traction mappings; nonexpansive mappings; implit iteration process; explicit iteration process; diffusion equation.

\section{Introduction}

Let $E$ be Banach space, and $k$ be a nonempty closed convex subset of $E$. Suppose that $T$ is a mapping from $K$ to $K$, and $F(T)$ is a set of fixed point of $T$ with $F(T) \neq \phi$. Assume that $J: E \rightarrow 2^{E}$ is regular dual mapping on $E$, and $J(x)=\left\{f \in E^{*} \mid<x, f>=\|x\| f\|\| f,\|=\| x \|\right\}, x \in E$.

As $E=H$ is Hilbert space, the internal product of $H$ is donate by the symbol $<\bullet, \bullet>$, and the norm of $H$ is designated by symbol $\|\bullet\|$.

Definition 1 Mapping $T: K \rightarrow K$ is said to be pseudo contraction if for arbitrary $x, y \in K$, there exits $j(x-y) \in J(x-y)$ such that $\left\langle T x-T y, j(x-y)>\leq\|x-y\|^{2}\right.$.

$T$ is said to be strong pseudo contraction if there is $k \in(0,1)$ such that $<T x-T y, j(x-y)>\leq k\|x-y\|^{2}$ for arbitrary $x, y \in K$. Definition 2 Mapping $T: K \rightarrow K$ is said to be nonexpanxive if for abitrary $x, y \in K$, there is $\|T x-T y\| \leq\|x-y\|$.

As we all know, that $T$ is pseudo contraction is equivalent to that for every $S>0$ and every $x, y \in K$, there is

$$
\|x-y\| \leq\|x-y+s[(I-T) x-(I-T) y]\|
$$

When $E=H$ is Hilbert space, $J: E \rightarrow 2^{E}$ is single value, and for abitrary $x, y \in K$, there Is

$$
<x-y, j(x-y)\rangle=<x-y, x-y>.
$$

Obviously, nonexpansive mapping is pseudo contraction.

\section{Lemmas and Methods}

Lemma $1^{[1,2]}$ Let $E$ be a real Banach space , and $K$ be nonempty closed convex subset of $E$. Assume that $T: K \rightarrow K$ is continuous strong pseudo constraction mapping. Then $T$ is unique fixed point in $K$.

Lemma2 Let $E$ be a real reflexive Banach space satisfying opial condition, and $K$ be a nonempty closed convex subset of $E$. Supposethat $T: K \rightarrow K$ is continuous strong pseudo constraction mapping . Then for abitrary $\left\{x_{n}\right\} \subset E$,

$x_{n}$ weakly converge to $y$, and $\left\|x_{n}-T x_{n}\right\| \rightarrow 0$. So there is $(I-T) x=0$.

Lemma $3^{[4]}$ Let $p>1, r>0$ be two certain real number, then Banach space is $(I-T) x=0$ if and only if there is a strictly

increasing continuous function $g:[0,+\infty) \rightarrow[0,+\infty), g(0)=0$, such that

$$
\|\lambda x+(1-\lambda) y\|^{p} \leq \lambda\|x\|^{p}+(1-\lambda)\|y\|^{p}-W_{p}(\lambda) g(\|x-y\|),
$$

for all $x, y \in B_{r}$, where $\lambda \in[0,1]$, and $B_{r}$ is a closed spheroid which center is origin and radius is $r$, and

$$
W_{p}(\lambda)=\lambda^{p}(1-\lambda)+\lambda(1-\lambda)^{p}
$$

Lemma 4 Let nonnegative real sequence $\left\{a_{n}\right\}$ satisfy the inequaltiy : $a_{n+1} \leq\left(1-\gamma_{n}\right) a_{n}+\delta_{n}, n \geq 0$, where $\gamma_{n} \in[0,1)$, $\sum_{n=1}^{\infty} \gamma_{n}=\infty, \lim _{n \rightarrow \infty} \frac{\delta_{n}}{\gamma_{n}}=0$ or $\sum_{n=1}^{\infty}\left|\delta_{n}\right|<+\infty$, then $\lim _{n \rightarrow \infty} a_{n}=0$. 
In Hilbert space, Moudaf ${ }^{[6]}$ has get strong convergence theorem of implicit iteration process of nonexpansive mapping, and $X u^{[7]}$ has improved and extended some relative results in Reference [7].

In this paper, by applying a new implicit iteration sequence $x_{n}=\alpha_{n} f\left(x_{n}\right)+\beta_{n} x_{n}+\gamma_{n} T x_{n}$, and explicit iterative sequence $y_{n+1}=\alpha_{n} f\left(y_{n}\right)+\beta_{n} y_{n}+\gamma_{n} T y_{n}$, we shall consider the problem involving the fixed point of strong pseudo constraction and nonexpansive mapping on closed convex set $\mathrm{K}$. When exact conditions are satisfied, $\left\{x_{n}\right\}$ and $\left\{y_{n}\right\}$ all strongly converge to the fixed point of $T$. When the conditions for $\left\{\alpha_{n}\right\}$ and $f$ in Reference[6],[7] are widened, and as $\beta_{n}=0$, we can obtain the iterative sequence in Reference [6],[7], and then we improve and extend some ralative results and obtain some equation of diffusion by applying the above results.

Let $T: K \rightarrow K$ be continuous pseudo constraction mapping, and $f: K \rightarrow K$ be continuous strong pseudo constraction mapping with constant $\alpha(0<\alpha<1)$. Suppose that $\alpha_{n}+\beta_{n}+\gamma_{n}=1$ for $\alpha_{n}, \beta_{n}, \gamma_{n} \in(0,1)$, and we stucture mapping $S_{n}: K \rightarrow K, S_{n} x=\alpha_{n} f(x)+\beta_{n} x+\gamma_{n} T x$. Then $S_{n}$ is continuous strong pseudo constraction mapping. By virtue of Lemma $1, S_{n}$ hasunique fixed point $x_{n}$, then we have

$$
x_{n}=S_{n} x_{n}=\alpha_{n} f\left(x_{n}\right)+\beta_{n} x_{n}+\gamma_{n} T x_{n}
$$

\section{Main Results}

Theorem 1 Let $E$ be a Hilbert space, and $K$ be a nonempty closed convex subset of $E$. Assume that $f: K \rightarrow K$ is continuous strong pseudo constraction mapping with constant $\alpha(0<\alpha<1)$, and $f$ is bounded on bounded set, and $T: K \rightarrow K$ is continuous pseudo constraction mapping .Then

(a) If $\sigma_{n}=\frac{\alpha_{n}}{1-\beta_{n}} \rightarrow 0$ or $\limsup _{n \rightarrow \infty} \sigma_{n}<1$, and there

is $p \in F(T)$ such that $\left\|f\left(x_{n}\right)-p\right\|^{2}-\left\|x_{n}-p\right\|^{2} \rightarrow 0$, then implict iterative sequence (2) strongly converges to the point of $F(T)$.

(b) If $T$ is nonexpansive mapping and $f$ is constraction mapping with constant $\alpha$,as $\frac{\left|\sigma_{n}-\sigma_{n-1}\right|}{\alpha_{n} \sigma_{n}} \rightarrow 0$ and $\sum \alpha_{n}=\infty$, the explicit iterative sequence $y_{n+1}=\alpha_{n} f\left(y_{n}\right)+\beta_{n} y_{n}+\gamma_{n} T y_{n}$ strongly converges to the point of $F(T)$.

\section{Proof.}

(a) Because $\forall p \in F(T)$,

$$
\left\|x_{n}-p\right\|^{2}
$$

$=\left\langle\alpha_{n}\left(f\left(x_{n}\right)-p\right)+\beta_{n}\left(x_{n}-p\right)+\gamma_{n}\left(T x_{n}-p\right), x_{n}-p\right\rangle$

$\leq \alpha \alpha_{n}\left\|x_{n}-p\right\|^{2}+\alpha_{n}\|f(p)-p\|\left\|x_{n}-p\right\|+\beta_{n}\left\|x_{n}-p\right\|^{2}+\gamma_{n}\left\|x_{n}-p\right\|^{2}$, we have $\left\|x_{n}-p\right\| \leq \frac{\alpha_{n}}{1-\alpha \alpha_{n}-\beta_{n}-\gamma_{n}}\|f(p)-p\|=\frac{\alpha_{n}}{1-\alpha}\|f(p)-p\|$.

Hence $\left\{x_{n}\right\},\left\{f\left(x_{n}\right)\right\},\left\{T x_{n}\right\}$ are bounded.

If $\sigma_{n}=\frac{\alpha_{n}}{1-\beta_{n}} \rightarrow 0$,then using formula (2),we can write $x_{n}=\sigma_{n} f\left(x_{n}\right)+\left(1-\sigma_{n}\right) T x_{n}$, and then we obtain

$$
\left\|x_{n}-T x_{n}\right\|=\sigma_{n}\left\|f\left(x_{n}\right)-T x_{n}\right\| \rightarrow 0 .
$$

If $\limsup \sigma_{n}<1$ and there is $p \in F(T)$ such that $n \rightarrow \infty$

$\left\|f\left(x_{n}\right)-p\right\|^{2}-\left\|x_{n}-p\right\| \rightarrow 0$, then by virtue of formula and Lemma 3 , we obtain

$$
\begin{aligned}
& \left\|x_{n}-p\right\|^{2} \\
\leq & \left\|x_{n}-p+\frac{1-\sigma_{n}}{2 \sigma_{n}}\left(x_{n}-T x_{n}\right)\right\|^{2} \\
= & \left\|x_{n}-p+\frac{1-\sigma_{n}}{2}\left(f\left(x_{n}\right)-T x_{n}\right)\right\|^{2} \\
= & \left\|\frac{1}{2}\left(f\left(x_{n}\right)-p\right)+\frac{1}{2}\left(x_{n}-p\right)\right\|^{2} \\
\leq & \frac{1}{2}\left\|f\left(x_{n}\right)-p\right\|^{2}+\frac{1}{2}\left\|x_{n}-p\right\|^{2}-\frac{1}{4} g\left(\left\|x_{n}-f\left(x_{n}\right)\right\|\right),
\end{aligned}
$$
and then

$\frac{1}{2} g\left(\left\|x_{n}-f\left(x_{n}\right)\right\|\right) \leq\left\|f\left(x_{n}\right)-p\right\|^{2}-\left\|x_{n}-p\right\|^{2} \rightarrow 0$.

So we have $\left\|x_{n}-f\left(x_{n}\right)\right\| \rightarrow 0$.

Whereas

$$
\left\|x_{n}-T x_{n}\right\|=\sigma_{n}\left\|f\left(x_{n}\right)-T x_{n}\right\|=\frac{\sigma_{n}}{1-\sigma_{n}}\left\|x_{n}-f\left(x_{n}\right)\right\| \rightarrow 0
$$

(4)

Because $\left\{x_{n}\right\}$ is bounded, and $E$ is Hilbert space, we have that $x_{n}$ weakly converge to $q \in K$. By virtue of formula 
(3)or (4) and Lemma 2, we have $q \in F(T)$.

Because

$\left\|x_{n}-q\right\|^{2}=<\sigma_{n}\left(f\left(x_{n}\right)-q\right)+\left(1-\sigma_{n}\right)\left(T x_{n}-q\right), x_{n}-q>$

$\leq \alpha \sigma_{n}\left\|x_{n}-q\right\|^{2}+\sigma_{n}<f(q)-q, x_{n}-q>+\left(1-\sigma_{n}\right)\left\|x_{n}-q\right\|^{2}$,

we obtain $\left\|x_{n}-q\right\|^{2} \leq \frac{1}{1-\alpha}<f(q)-q, x_{n}-q>$. Since $x_{n}$ weakly converges to $q, x_{n}$ strongly converges to $q \in F(T)$.

(b) Because

$\left\|x_{n}-x_{n-1}\right\|$

$=\left\|\sigma_{n} f\left(x_{n}\right)+\left(1-\sigma_{n}\right) T x_{n}-\sigma_{n-1} f\left(x_{n-1}\right)-\left(1-\sigma_{n}\right) T x_{n-1}\right\|$

$\leq \sigma_{n} \alpha\left\|x_{n}-x_{n-1}\right\|+\left|\sigma_{n}-\sigma_{n-1}\right|\left\|f\left(x_{n-1}\right)\right\|+\left(1-\sigma_{n}\right)\left\|x_{n}-x_{n-1}\right\|+\left|\sigma_{n}-\sigma_{n-1}\right|\left\|T x_{n-1}\right\|$,

we obtain

$$
\left\|x_{n}-x_{n-1}\right\| \leq \frac{\left|\sigma_{n}-\sigma_{n-1}\right|}{\sigma_{n}(1-\alpha)} M
$$

where $\left\|f\left(x_{n-1}\right)\right\| \leq \frac{M}{2},\left\|T x_{n-1}\right\| \leq \frac{M}{2}$.

$$
\left\|y_{n+1}-y_{n}\right\|
$$

$=\left\|\alpha_{n} f\left(y_{n}\right)+\beta_{n} y_{n}+\gamma_{n} T y_{n}-\alpha_{n} f\left(x_{n}\right)-\beta_{n} x_{n}-\gamma_{n} T x_{n}\right\|$

$\leq \alpha \alpha_{n}\left\|y_{n}-x_{n}\right\|+\beta_{n}\left\|y_{n}-x_{n}\right\|+\gamma_{n}\left\|y_{n}-x_{n}\right\|=\left[1-\alpha_{n}(1-\alpha)\right]\left\|y_{n}-x_{n}\right\|$

$\leq\left[1-\alpha_{n}(1-\alpha)\right]\left\|y_{n}-x_{n-1}\right\|+\left[1-\alpha_{n}(1-\alpha)\right]\left\|x_{n}-x_{n-1}\right\|$

Since $\sum \alpha_{n}=\infty, \frac{\left|\sigma_{n}-\sigma_{n-1}\right|}{\alpha_{n} \sigma_{n}(1-\alpha)} \rightarrow 0$, formula (5) and

Lemma 4, we obtain $\left\|y_{n+1}-x_{n}\right\| \rightarrow 0$.

Hence we have $\left\|y_{n+1}-q\right\| \leq\left\|y_{n+1}-x_{n}\right\|+\left\|x_{n}-q\right\| \rightarrow 0$, which means that $\left\{y_{n}\right\}$ strongly converges to $q \in F(T)$.

Note.

Theorem 1 improves and extends some relative results in Reference [6] and [7].

As follows, we will discuss iterative solution of some response diffusion equation .

Let $E=L^{2}(I)=\left\{x(t, s) \mid(t, s) \in I, x(t, s)\right.$ and $x^{2}(t, s)$ are Lebsgue intergrable on $\mathrm{I}\}$, where $I=[a, b] \times[c, d]$, and $\forall x, y \in E$

,we define $\langle x, y\rangle=\iint_{I} x(t, s) y(t, s) d t d s$.Then $E$ is Hilbert

space, and $\|x\|^{2}=<x, x>=\iint_{I} x^{2}(t, s) d t d s$,

$$
<y, j(x)>=<y, x>, \forall x, y \in E .
$$

Consider the problem involving solution of some first order

$$
\left\{\begin{array}{l}
\frac{\partial x}{\partial t}=-u_{0} \frac{\partial x}{\partial s}-x G x-h x, \\
x(s, 0)=x_{0}(s), x(0, t)=x_{1}(t),
\end{array}\right.
$$

where $G$ is continuous mapping on $E$, and $u_{0} \geq 0$ is constant, and $h=h(t, s) \geq 0$.

This problem is equivalent to the integral equation as follows:

$\int_{0}^{x} x(t, s) d s+u_{0} \int_{0}^{t} x(t, s) d t+\int_{0}^{t} \int_{0}^{s} h(t, s) x(t, s) d t d s+\int_{0}^{t} \int_{0}^{s} x G x d t d s$

$-\int_{0}^{s} x_{0}(s) d s-u_{0} \int_{0}^{t} x_{1}(t) d t=0$

Let $K=\{x \in E \mid x(t, s)$ is continuous function on $I\}$, then $K$ is nonempty closed convex subset of $E$.

Let $H: K \rightarrow K$.

$H x=u_{0} \int_{0}^{t} x(t, s) d t+\int_{0}^{s} x(t, s) d s+\int_{0}^{t} \int_{0}^{s} h(t-x) x(t-s) d t d s+\int_{0}^{t} \int_{0}^{s} x G x d t d s$ $-\int_{0}^{s} x_{0}(s) d s-u_{0} \int_{0}^{t} x_{1}(t) d t$

If $G$ satisfies (A): $\forall x, y \in K, x G x \leq y G y$, then let $T: K \rightarrow K, T x=-H x+x$.

If $G$ satisfies (B) : there is $L_{1}>0$ such that $\|x G x-y G y\| \leq L_{1}\|x-y\|$ for abitrary $x, y \in K$. Then $H$ is Lipschitz mapping on $K$, and then we have $L>0$ such that $\forall x, y \in K,\|H x-H y\| \leq L\|x-y\|$.

Let $H_{1}=\frac{2}{L} H, T_{1}: K \rightarrow K, T_{1} x=-H_{1} x+x$.

Theorem 2 Let integral equation (7) has solution, then

(i) If $G$ satisfies (A), when $\sigma_{n}=\frac{\alpha_{n}}{1-\beta_{n}} \rightarrow 0$ or $\limsup \sigma_{n}<1$, and there is $p \in F(T)$ such that $\left\|f\left(x_{n}\right)-p\right\|^{2}-\left\|x_{n}-p\right\|^{2} \rightarrow 0$, Implicit iterative sequence $x_{n}=\alpha_{n} f\left(x_{n}\right)+\beta_{n} x_{n}+\gamma_{n} T x_{n}$ strongly converges to the fixed point of $T$ which is solution of equation (7).

(ii) If $G$ satisfies (B ), when $\sum \alpha_{n}=\infty$ and $\frac{\left|\sigma_{n}-\sigma_{n-1}\right|}{\alpha_{n} \sigma_{n}} \rightarrow 0$, explicit iterative sequence $y_{n+1}=\alpha_{n} f\left(y_{n}\right)+\beta_{n} y_{n}+\gamma_{n} T y_{n}$ strongly converges to the fixed point of $T$ which is solution of equation (7).

Proof.

(i) Now, $\forall x, y \in K,(H x-H y)(x-y)$ is nonnegative on diffusion equation: 
$E_{1}=\{(t, s) \in I \mid x-y \leq 0\}$ and $E_{2}=\{(t, s) \in I \mid x-y \geq 0\}$.

Then we have $<H x-H y, x-y>\geq 0$, that is said that

$$
T \text { is }
$$

pseudo constraction mapping on $K$. Using Theorem 1 , we obtain the result .

(ii) Now $\quad, \quad \forall x, y \in K,\left\|H_{1} x-H_{1} y\right\| \leq 2\|x-y\|$

$$
\begin{aligned}
& \left(T_{1} x-T_{1} y\right)^{2} \\
= & {\left[-H_{1} x+x-\left(-H_{1} y+y\right)\right]^{2} } \\
= & \left.\left(H_{1} x-H_{1} y\right)^{2}-2 y-x\right)\left(H_{1} y-H_{1} x\right)+(x-y)^{2} \\
= & \left(H_{1} y-H_{1} x\right)^{2}-2|y-x|\left|H_{1} y-H_{1} x\right|+(x-y)^{2} \\
= & \left(H_{1} y-H_{1} x\right)\left(\left|H_{1} y-H_{1} x\right|-2|y-x|\right)+(x-y)^{2}
\end{aligned}
$$

If $\left|H_{1} y-H_{1} x\right| \leq 2|y-x|$, then we obtain

$$
\left(T_{1} x-T_{1} y\right)^{2} \leq(x-y)^{2} .
$$

If $\left|H_{1} y-H_{1} x\right| \geq 2|y-x| \geq 0$, then we obtain

$$
\begin{aligned}
\left(T_{1} x-T_{1} y\right)^{2} & \leq\left(\left|H_{1} y-H_{1} x\right|+2|y-x|\right)\left(\left|H_{1} y-H_{1} x\right|-2|y-x|\right)+(x-y)^{2} \\
& \left.=\left(H_{1} y-H_{1} x\right)^{2}-4 x+y\right)^{2}+(x-y)^{2} .
\end{aligned}
$$

Hence, by virtue of formula (8), we have

$$
\left\|T_{1} x-T_{1} y\right\|^{2} \leq\|x-y\|^{2} .
$$

That is said that $T_{1}$ is nonexpansive mapping on $K$. Using Theorem 1, we obtain the result.

Therefore, through improving some corresponding conditions in literature [6],[7], and extending some recent relevent results, Theorem 1 was established. Theorem 1 is a strong convergence theorem of some implicit iteration process for pesudocon-traction mappings and explicit iteration process for nonexpansive mappings. By applying Theorem 1, the iterative solution for some equation of response diffusion was obtained, Theorem 2 was established.

\section{REFERENCES}

[1] Deimling K. zeros of accretive operators [J].Manuscripta Math, 1974,13(4):365-374.

[2] Chang S S. Cho Y J. Zhou H Y. Iterutive methods for nonlinear operator Equation in Banach space [M].New York: Science publishers, 2002.

[3] Zhou H Y. Convergence theorems of common fixed points for a finite family of Lipschitzian pseudocontractions inBanachspaces [J].Nonlinear Anal ,2008,68(10):2977-2983

[4] Xu H K. Inequalities in Banach space with applications, Nonlinear Anal TMA ,1991,16(2):1127-1138.

[5] Xu H K. Iterative algorithms for nonlinear operator [J]. J London Math Soc,2002,66:240-256.

[6] Moudafi A. Viscosity approximation methods for fixedpoints problems [J]. J Math Anal Appl.2000,241:46-55.

[7] Xu H K. Viscosity approximation methods for nonexpa nsive mapping [J]. J Math Anal Appl,2004,298:279-291. 